Fast jeder zehnte deutsche Haushalt beschäftigt nach Schätzungen regelmäßig oder gelegentlich Putzhilfen. Sie arbeiten nicht nur bei „den Reichen“, sondern auch in Haushalten mit eher niedrigem Einkommen, gerade auch bei älteren Menschen, wenn auch dann oft nur stundenweise. In der Statistik tauchen jedoch nur 40.000 Haushaltshilfen als sozialversicherungspflichtige Beschäftigte auf, zuzüglich 128.000 legale Minijobs. Dazwischen liegt die Grauzone der Schwarzarbeit. Wie viele der Haushaltshilfen aus dem Ausland kommen, weiß niemand genau, aber es dürften viele sein. Ihnen widmete sich Prof. Dr. Maria Rerrich von der Fachhochschule München in einer qualitativen Studie, die kürzlich erschienen ist („Die ganze Welt zu Hause - Cosmobile Putzfrauen in privaten Haushalten“, Hamburger Edition). Aus diesem Anlass war die Autorin am 6.12.06 zu Gast zu einer Lesung an der Fachhochschule Frankfurt am Main; eingeladen hatte das Frauenforschungskolloquium des Fachbereichs Soziale Arbeit und Gesundheit, das gemeinsame Frauenforschungszentrum der Hessischen Fachhochschulen (gFFZ) und das CorneliaGoethe-Centrum der Universität Frankfurt am Main. Rerrich konfrontierte die ZuhörerInnen mit dem Phänomen einer Schattenwirtschaft mit feudalen Zügen, in der moderne Dienstboten kaum mehr Rechte haben als jene des 19. Jahrhunderts - und dies relativ unbeachtet von Öffentlichkeit und Politik. Wenn sie ohne legalen Aufenthaltsstatus im Land leben und ohne Erlaubnis arbeiten, befinden sie sich in extrem ungeschützten Arbeitsverhältnissen: Feierabend, Freizeit, Urlaub sind vom Wohlwollen der privaten Arbeitgeber abhängig, ganz zu schweigen von der Frage der Lohnfortzahlung im Krankheitsfall. Weil in der Regel in mehreren Haushalten sowie meist ohne Schutzmaßnahmen (wie sie öffentliche Reinigungsfirmen und Institutionen einhalten müssen) gearbeitet wird, erleiden diese Arbeiterinnen über kurz oder lang schmerzhafte Gesundheitsschäden, für die es wiederum nur informelle medizinische Anlaufstellen gibt. Maria Rerrich hat mit solchen „cosmobilen Putzfrauen“ in München und Hamburg ausführlich gesprochen. Ihre Geschichten müssen aus vielen Gründen nachdenklich machen: Die Erzählerinnen fühlen sich nicht als Opfer, sondern sind voller Zuversicht und positiver Selbsteinschätzung. Sie kommen aus den armen Ländern der Welt nach Deutschland, um hier mehr Geld zu verdienen als es ihnen in ihren Heimatländern möglich ist. Dieses Geld brauchen sie, um ihre zurückgelassenen Familien zu unterstützen. Zu Krisen kommt es daher, wenn sich zeigt, dass der Preis für die Familiensicherung zu hoch war - wenn die große räumliche Entfernung, die lange Trennung ihren Tribut fordern und das Familienprojekt letztlich scheitern lassen. Die Geschichten zeigen auch: Es sind nicht die Ärmsten der Armen, die kommen, sondern oftmals gut qualifizierte Frauen. Das Putzen im Haushalt müssen sie manches Mal erst mühsam an ihren neuen deutschen Arbeitsstellen lernen. Dennoch bringen sie aufgrund ihres Ausbildungsniveaus Schlüsselqualifikationen mit, die sie für den Einsatz in den Privathaushalten besonders attraktiv machen: Kommunikations- und Beziehungsfähigkeit, Organisations- und Planungsvermögen. Die deutschen Haushalte profitieren von dieser Überqualifizierung, für die Herkunftsländer bedeutet diese Ressourcenabschöpfung jedoch ein enormes Problem, denn dort fehlen die dringend benötigten qualifizierten Kräfte. Rerrich verbindet ihre Studie mit einem energischen Plädoyer für eine sozialpolitische Debatte um private Haushaltstätigkeiten. Die Tatsache, dass so viele Haushalte Putzfrauen „schwarz“ beschäftigen, verweist auf ein ungelöstes, bislang erfolgreich privatisiertes strukturelles Problem - nämlich die Frage der Organisation der individuellen Reproduktion. Die Autorin sieht zwei tragfähige Alternativen: Es ist entweder darauf hinzuwirken, dass die anfallenden Arbeiten in den deutschen Haushalten von den Einheimischen leichter selbst verrichtet werden können, ob nun bezahlt oder unbezahlt, oder es ist dafür zu sorgen, dass die Arbeits- und Bürgerrechte für die Menschen gesichert sind, die aus dem Ausland kommen, um diese Arbeiten zu verrichten. Dass der offensichtliche Bedarf an Dienstleistungen im Privaten von einer unsichtbaren Gruppe von Frauen unter fragwürdigen Bedingungen bewältigt wird, ist jedenfalls ein unhaltbarer Zustand. Rerrichs Studie sind von daher viele LeserInnen zu wünschen.

\section{Cosmobile Putzfrauen}

